\title{
Treatment of congenital fibrinogen deficiency: overview and recent findings
}

\author{
This article was published in the following Dove Press journal: \\ Vascular Health and Risk Management \\ 3 October 2009 \\ Number of times this article has been viewed
}

\section{Konstantinos Tziomalos Sofia Vakalopoulou Vassilios Perifanis Vassilia Garipidou}

Second Propedeutic Department of Internal Medicine, Medical School, Aristotle University of Thessaloniki, Hippokration Hospital, Thessaloniki, Greece
Correspondence: Konstantinos Tziomalos 63 Solonos Street, Thessaloniki, 54248, Greece

Tel +31 2310823487

Email ktziomalos@yahoo.com
Abstract: Afibrinogenemia is a rare bleeding disorder with an estimated prevalence of $1: 1,000,000$. It is an autosomal recessive disease resulting from mutations in any of the 3 genes that encode the 3 polypeptide chains of fibrinogen and are located on the long arm of chromosome 4. Spontaneous bleeding, bleeding after minor trauma and excessive bleeding during interventional procedures are the principal manifestations. We review the management of afibrinogenemia. Replacement therapy is the mainstay of treatment of bleeding episodes in these patients and plasma-derived fibrinogen concentrate is the agent of choice. Cryoprecipitate and fresh frozen plasma are alternative treatments that should be used only when fibrinogen concentrate is not available. Secondary prophylactic treatment may be considered after life-threatening bleeding whereas primary prophylactic treatment is not currently recommended. We also discuss alternative treatment options and the management of surgery, pregnancy and thrombosis in these patients. The development of new tests to identify higher risk patients and of safer replacement therapy will improve the management of afibrinogenemia in the future.

Keywords: afibrinogenemia, fibrinogen concentrate, cryoprecipitate, fresh frozen plasma, rare bleeding disorders

\section{Introduction}

Afibrinogenemia is a rare bleeding disorder with an estimated prevalence of $1: 1,000,000 .^{1,2}$ It is an autosomal recessive disease and most patients are commonly descendent of consanguineous marriages. ${ }^{1-3}$ Afibrinogenemia results from mutations in any of the 3 genes (FGA, FGB and FGG) that encode the 3 polypeptide chains of fibrinogen $(\mathrm{A} \alpha, \mathrm{B} \beta$ and $\gamma$ ) and are located in a $50 \mathrm{~kb}$ region on $4 \mathrm{q} 31.3{ }^{4}$ These mutations affect the synthesis, assembly, intracellular processing, stability or secretion of fibrinogen. ${ }^{4}$ Fibrinogen plays an important role in clot formation through its conversion to fibrin by the action of thrombin. ${ }^{5,6}$ It is also important in primary hemostasis since it contributes to platelet aggregation by binding to glycoprotein IIb/IIIa on the activated platelet surface. ${ }^{5,6}$ The diagnosis of afibrinogenemia is based on the presence of prolonged prothrombin, thrombin, reptilase and activated partial thromboplastin time, undetectable functional fibrinogen and absence or trace amounts of immunoreactive fibrinogen. ${ }^{5}$ Platelet adhesion and ADP-induced platelet aggregation are also impaired in these patients whereas thrombin- and collagen-stimulated platelet aggregation is normal. ${ }^{7-10}$ submit your manuscript | www.dovepress.com

Dovepress 
We review the principal clinical manifestations and the management of patients with afibrinogenemia. We also discuss the case for prophylactic treatment and the management of pregnancy in these patients.

\section{Clinical manifestations}

The commonest manifestations of afibrinogenemia are umbilical stump bleeding (which can be life threatening) and bleeding from mucosal surfaces, particularly menorrhagia, epistaxis and bleeding in the oral cavity. ${ }^{3}$ Musculoskeletal bleeding (including hemarthroses) is observed in around half of the patients ${ }^{3,11}$ and in some series it was more frequent than mucosal bleeding. ${ }^{12}$ These discrepant results on the relative frequency of bleeding complications are partly due to the small number of patients included in the reported series. ${ }^{3,12}$ Bleeding from the gastrointestinal and urinary tract is less frequent ${ }^{3}$ whereas intracranial bleeding is rare. ${ }^{3,12-15}$ However, recurrent episodes of intracranial hemorrhage have been reported. ${ }^{12,14-16}$ Hemoperitoneum and hemorrhagic corpus luteum following the rupture of a follicle during ovulation are other rare manifestations. ${ }^{13,17-20}$ The bleeding tendency is highly variable in afibrinogenemia (ranging between very few up to several episodes/year) even among patients with the same mutation..$^{5,12,21}$ The presence of modifier genes (yet unidentified) or the coexistence of thrombophilic disorders has been implicated as an explanation for this variability. ${ }^{21}$ Besides spontaneous bleeding, bleeding after minor trauma and excessive bleeding during interventional procedures are other principal manifestations of afibrinogenemia. ${ }^{2,3,11}$

Paradoxically, spontaneous thrombotic complications have also been reported in patients with afibrinogenemia, including thrombosis in peripheral arteries and in cerebral and hepatic veins. ${ }^{3,22,23}$ Fibrin down-regulates thrombin generation by reducing prothrombin activation and also binds and sequesters thrombin. ${ }^{24,25}$ This antithrombin activity of fibrin (antithrombin I) is absent in patients with afibrinogenemia, who exhibit increased prothrombin activation and thrombin generation. ${ }^{7,24,25}$ In addition, free thrombin may stimulate platelets to release several growth factors that induce vascular smooth muscle cell proliferation and intimal hyperplasia. ${ }^{26}$ All these abnormalities may contribute to the development of thrombotic complications. ${ }^{7,24-26}$ Ex vivo studies also suggested that larger and loosely packed platelet thrombi are formed in the absence of fibrinogen. ${ }^{27}$ In some patients, the coexistence of thrombophilic disorders (eg, protein C deficiency, hepatitis C infection with mixed cryoglobulinemia and anticardiolipin antibodies) might also contribute to thrombosis. ${ }^{28,29}$
Fibrinogen also plays a role in wound healing, with reports of impaired wound healing following surgery and slow-healing leg ulcers in patients with afibrinogenemia. ${ }^{3,30,31}$ Spontaneous splenic rupture is another rare manifestation. ${ }^{13,32-34}$

\section{Treatment}

\section{Replacement therapy}

Replacement therapy is the mainstay of treatment of bleeding episodes in patients with afibrinogenemia and includes plasma-derived fibrinogen concentrate, cryoprecipitate and fresh frozen plasma (FFP). ${ }^{5,35,36}$ The quantity of fibrinogen required can be calculated as follows:

$$
\begin{aligned}
\operatorname{Dose}(g)= & 0.07 * \text { desired increment }\left(\frac{g}{L}\right) *(1-\text { hematocrit }) \\
& * \text { patient weight }(\mathrm{kg}) .{ }^{36}
\end{aligned}
$$

Treatment should be administered daily or every other day based on the indication, response, potential fibrinogen consumption and monitoring of fibrinogen activity. ${ }^{5,36}$ Since the half-life of fibrinogen is approximately 4 days, alternate day administration should be sufficient in the absence of consumption. ${ }^{36}$ For spontaneous bleeding, recommended target fibrinogen levels are $>1 \mathrm{~g} / \mathrm{L}$ until hemostasis is achieved and $>0.5 \mathrm{~g} / \mathrm{L}$ until the bleeding surface is completely healed. ${ }^{5,36}$ However, many physicians appear to aim at higher fibrinogen levels possibly because of concerns about achieving hemostasis at lower levels; however, this strategy might increase the risk of thrombosis (see below). ${ }^{12}$

Fibrinogen concentrate is the replacement therapy of choice in patients with afibrinogenemia. ${ }^{35,36}$ Its main advantage over other replacement therapies is that it is virally inactivated; other advantages are a smaller infusion volume and a lower risk of allergic reactions. ${ }^{2}$ Viral inactivation of existing fibrinogen concentrates is performed either with solvent detergent exposure (alone or in combination with dry heating and/or nanofiltration) or with pasteurization. ${ }^{21}$ However, non-lipid-enveloped viruses such as parvovirus B19 and hepatitis A virus may survive in currently used inactivation processes. ${ }^{35,37,38}$ Moreover, since all fibrinogen concentrates are plasma-derived, there is a theoretical potential for transmission of new non-viral pathogens (eg, prions). ${ }^{21,35}$ There are 5 fibrinogen concentrates currently available, Haemocomplettan ${ }^{\circledR}$ P (CSL Behring, Marburg, Germany), Clottagen ${ }^{\circledR}$ and FIBRINOGENE T $1{ }^{\circledR}$ (LFB, Les Ullis, France), Fibrinogen $\mathrm{HT}^{\circledR}$ (Benesis, Osaka, Japan) and FibroRAAS $^{\circledR}$ (Shangai RAAS, Shangai, China). ${ }^{21,39}$ These products are not available or licensed in all countries and there are no studies directly comparing their efficacy. In an 
open uncontrolled study, the half-life of Haemocomplettan $\mathrm{P}$ was between 2.5 and 5.2 days, ie, similar to the half-life of fibrinogen in normal individuals. ${ }^{40}$ A dose of $1 \mathrm{mg} / \mathrm{kg}$ Haemocomplettan $\mathrm{P}$ increased fibrinogen concentration by $0.015 \mathrm{~g} / \mathrm{L}$ and normalized coagulation parameters during the day of infusion. ${ }^{40}$ In another open, uncontrolled, retrospective study, Haemocomplettan $\mathrm{P}$ was effective in both the treatment of spontaneous bleeding episodes and as prophylaxis before surgical procedures or against spontaneous bleeding. ${ }^{41}$ The median post-infusion fibrinogen levels were $1.45 \mathrm{~g} / \mathrm{L}$ and a reduction in both thrombin and activated partial thromboplastin time was observed after infusion. ${ }^{41}$ The median single and total doses per episode were 2.0 and 4.0 g per patient, respectively, and the median duration of treatment was 1 day. ${ }^{41}$ One patient given prophylactic infusions developed an anaphylactic reaction and another patient undergoing osteosynthesis developed deep vein thrombosis and pulmonary embolism despite postoperative prophylaxis with heparin. ${ }^{41}$ No other side effects were reported. ${ }^{41}$ In a more recent open, uncontrolled, prospective study, target fibrinogen levels were achieved within 1 hour after the infusion of FIBRINOGENE T1 and the between-patient variability in pharmacokinetics was very small. ${ }^{39}$ The mean half-life of fibrinogen was 3.4 days and coagulation parameters were normalized for 6 to 10 days after the infusion. ${ }^{39}$ Administration of fibrinogen concentrate was also shown to correct platelet adhesion and ADP-induced platelet aggregation even at fibrinogen levels as low as $10 \%$ of normal. ${ }^{8}$

Cryoprecipitate and FFP represent alternative replacement therapies and should be given only in emergencies when fibrinogen concentrate is not available. ${ }^{21,35,36}$ Cryoprecipitate and FFP can be either single donor or pooled. ${ }^{35}$ Single donor cryoprecipitate and FFP can be either virally inactivated (using methylene blue/visible light treatment) or non-virally inactivated. ${ }^{35}$ Pooled cryoprecipitate is non-virally inactivated whereas both virally inactivated (with solvent detergent treatment) and non-virally inactivated pooled FFP exist. ${ }^{35}$ However, the single-step viral inactivation of cryoprecipitate and FFP is considered inferior (particularly against non lipid-enveloped viruses) compared with fibrinogen concentrates that have undergone 2 viral inactivation steps. ${ }^{35}$ The amount of fibrinogen in cryoprecipitate and FFP varies but is lower than in fibrinogen concentrates. ${ }^{12}$ Cryoprecipitate is enriched in fibrinogen but methylene blue/visible light treatment of cryoprecipitate reduces fibrinogen activity by $40 \% .{ }^{35}$ According to current guidelines, virally inactivated FFP should be preferred to non-virally inactivated cryoprecipitate unless volume overload poses unacceptable risks. ${ }^{35}$

Thrombotic complications have been reported in patients with afibrinogenemia following replacement therapy, including ischemic foot lesions, ischemic stroke, renal and ovarian vein thrombosis, deep venous thrombosis and pulmonary embolism. ${ }^{12,13,15,41-44}$ Patients with afibrinogenemia who also suffer from inherited thrombophilic disorders (eg, protein $\mathrm{C}$ deficiency) appear to be at higher risk for thrombosis after replacement therapy. ${ }^{43}$ In addition, the risk might be greater when cryoprecipitate is used since the latter contains substantial quantities of other coagulation factors (particularly von Willebrand factor and factor VIII). ${ }^{21}$ In patients with afibrinogenemia who exhibited thrombosis after cryoprecipitate treatment, switching to fibrinogen concentrate reduced the risk of recurrent thrombosis. ${ }^{15}$ The management of thrombotic complications in patients with afibrinogenemia is problematic because of their bleeding tendency. Some authors proposed the concomitant administration of replacement therapy with low-dose heparin. ${ }^{43}$ Others recommend use of compression stockings and low molecular weight heparin in patients with a history of thrombosis who are undergoing surgery. ${ }^{21}$ In patients who develop thrombotic complications following replacement therapy, some authors continue the latter if indicated and co-administer low-molecular-weight or unfractionated heparin. ${ }^{13,15}$ In patients who develop recurrent thrombotic complications despite treatment with heparin and aspirin, treatment with lepirudin, a direct thrombin inhibitor, appears to be effective. ${ }^{45}$

In contrast to hemophilia, development of fibrinogen inhibitors following replacement therapy has been very rarely reported in patients with afibrinogenemia. ${ }^{46,47}$ It was suggested that the infrequent development of fibrinogen inhibitor is due to the presence of trace amounts of circulating fibrinogen. ${ }^{21}$

\section{Other treatments}

Fibrin glue can be used in dental extractions and in superficial wounds. ${ }^{36}$ Tranexamic acid inhibits fibrinolysis by binding plasmin and might also be useful in the treatment of mucosal bleeding. ${ }^{35,36}$ It can be administered orally, intravenously or as a mouth wash. ${ }^{35}$ However, it might increase the risk of thrombosis and should be used with caution in patients with a history of thrombosis. ${ }^{36}$ It should also be avoided in pregnant women, in patients who had recently undergone surgery or are immobilized and in cases of hematuria. ${ }^{35,36}$ Tranexamic acid should be given at a lower dose in patients with renal failure. ${ }^{35}$ 
In patients with splenic rupture, splenectomy after administering fibrinogen concentrate appears to be the treatment of choice. ${ }^{32,48}$ Conservative management with replacement therapy aiming at fibrinogen levels $>1 \mathrm{~g} / \mathrm{L}$ has been reported in patients who are stable after fluid replacement. ${ }^{34}$ However, these patients appear to be at increased risk of recurrent splenic rupture. ${ }^{48}$

In patients with severe liver disease mandating liver transplantation, the latter also will cure afibrinogenemia since fibrinogen is synthesized in the liver. ${ }^{21,22}$

\section{Prevention}

Before surgery, fibrinogen levels should be raised to $1 \mathrm{~g} / \mathrm{L}$ and maintained at this level until hemostasis is established and above $0.5 \mathrm{~g} / \mathrm{L}$ until complete wound healing. ${ }^{36}$ However, many physicians appear to aim at higher fibrinogen levels. ${ }^{12}$ All invasive procedures in patients with afibrinogenemia should take place in centers directly served by a hemophilia center. $^{36}$

Prophylactic infusion of fibrinogen concentrate or cryoprecipitate has been reported, particularly after life-threatening bleeding episodes. ${ }^{12-15,49}$ Most patients are treated with weekly infusions but treatment every 2 weeks or once a month has also been used. ${ }^{12}$ However, the frequency of bleeding in an observational study was similar in patients treated on-demand and in those given prophylactic infusions. ${ }^{12}$ In the same study, more than half of the patients on prophylactic infusion experienced breakthrough bleeding, including intracranial hemorrhage. ${ }^{12}$ According to current guidelines, secondary prophylactic treatment may be considered after lifethreatening bleeding (eg intracranial bleeding) with a target fibrinogen level of $0.5 \mathrm{~g} / \mathrm{L} .{ }^{36}$ Primary prophylactic infusion is not recommended because of the risk of transmission of infectious diseases and development of inhibitors, allergic reactions and thrombotic complications. . $^{5,36}$

Estrogen-progesterone combination can be used as prophylaxis in patients with menorrhagia..$^{5,22,36,49,50}$ Oral contraceptives also appear to be effective for the prevention of follicle rupture-induced hemoperitoneum. ${ }^{18,19}$ However, oral contraceptives might increase the risk of thrombosis. ${ }^{22,51}$ Antifibrinolytic agents (eg, tranexamic acid and aminocaproic acid) can be used as prophylaxis before dental procedures. ${ }^{5,36}$ Intramuscular injections should be avoided in patients with afibrinogenemia. ${ }^{5}$ Patients should be vaccinated against hepatitis $\mathrm{A}$ and $\mathrm{B}$ by subcutaneous injection. ${ }^{36}$ Prenatal diagnosis is possible when both parents are known heterozygotes for afibrinogenemia mutations or already have an affected child. ${ }^{52}$

\section{Pregnancy}

Fibrinogen appears to contribute to placental implantation. ${ }^{53}$ Accordingly, there is an increased risk of spontaneous abortion in patients with afibrinogenemia, which occurs at 6 to 8 weeks of gestation if no replacement therapy is given. ${ }^{3,12,53-56}$ Patients with afibrinogenemia can have successful pregnancies if treated with fibrinogen concentrate or cryoprecipitate. ${ }^{3,13,49,53,55,57,58}$ However, preterm delivery appears to be more frequent in these women despite replacement therapy. ${ }^{53}$ Placental abruption has also been reported despite the administration of fibrinogen concentrate or cryoprecipitate..$^{13,58}$

Replacement therapy should be instituted as soon as possible in pregnancy in order to prevent fetal loss, probably from the 5th week of gestation. ${ }^{5,36,53}$ It should be continued throughout the pregnancy aiming at fibrinogen levels $>1.0 \mathrm{~g} / \mathrm{L} .{ }^{36}$ Hemorrhagic complications have been reported with lower fibrinogen levels. ${ }^{49}$ However, thrombotic complications have also been reported during replacement therapy in pregnancy. ${ }^{13}$ It appears that fibrinogen requirements increase as pregnancy progresses. ${ }^{13,53}$ During labor, some authors recommend higher levels $(>2.0 \mathrm{~g} / \mathrm{L})$ to prevent placental abruption. ${ }^{53}$ Both vaginal delivery and cesarean sections have been reported. ${ }^{53,59}$ During puerperium, lower fibrinogen levels appear to be sufficient to prevent bleeding. ${ }^{53}$ Pregnancy should be managed in obstetric departments of hospitals that have a hemophilia center. ${ }^{36}$

\section{Conclusions}

Even though afibrinogenemia is a rare disorder, it might acquire greater importance in the future because of the immigration of Islamic populations where this disorder is more prevalent due to consanguineous marriages. ${ }^{60}$ Patients with afibrinogenemia should be referred to and registered with a hemophilia center. ${ }^{36}$ The development of new tests to identify higher risk patients and of safer replacement therapy will improve the management of these patients in the future.

\section{Disclosures}

The authors have no conflicts of interest to declare.

\section{References}

1. Acharya SS, Coughlin A, Dimichele DM. Rare Bleeding Disorder Registry: deficiencies of factors II, V, VII, X, XIII, fibrinogen and dysfibrinogenemias. J Thromb Haemost. 2004;2(2):248-256.

2. Mannucci PM, Duga S, Peyvandi F. Recessively inherited coagulation disorders. Blood. 2004;104(5):1243-1252.

3. Lak M, Keihani M, Elahi F, Peyvandi F, Mannucci PM. Bleeding and thrombosis in 55 patients with inherited afibrinogenaemia. $\mathrm{Br} J$ Haematol. 1999;107(1):204-206. 
4. Asselta R, Duga S, Tenchini ML. The molecular basis of quantitative fibrinogen disorders. J Thromb Haemost. 2006;4(10):2115-2129.

5. Acharya SS, Dimichele DM. Rare inherited disorders of fibrinogen. Haemophilia. 2008;14(6):1151-1158.

6. Castaman G. Prophylaxis of bleeding episodes and surgical interventions in patients with rare inherited coagulation disorders. Blood Transfus. 2008;6(Suppl 2):s39-s44.

7. Dupuy E, Soria C, Molho P, et al. Embolized ischemic lesions of toes in an afibrinogenemic patient: possible relevance to in vivo circulating thrombin. Thromb Res. 2001;102(3):211-219.

8. Reininger AJ, Reininger CB, Spannagl M, et al. Effect of fibrinogen substitution in afibrinogenemia on hemorheology and platelet function. Thromb Haemost. 1995;74(3):853-858.

9. De Marco L, Girolami A, Zimmerman TS, Ruggeri ZM. von Willebrand factor interaction with the glycoprotein IIb/IIa complex. Its role in platelet function as demonstrated in patients with congenital afibrinogenemia. J Clin Invest. 1986;77(4):1272-1277.

10. Soria J, Soria C, Borg JY, et al. Platelet aggregation occurs in congenital afibrinogenaemia despite the absence of fibrinogen or its fragments in plasma and platelets, as demonstrated by immunoenzymology. $\mathrm{Br} J$ Haematol. 1985;60(3):503-514.

11. Vakalopoulou S, Rizopoulou D, Zafiriadou E, et al. Management of acute bleeding in a patient with congenital afibrinogenaemia. Haemophilia. 2006;12(6):676-678.

12. Peyvandi F, Haertel S, Knaub S, Mannucci PM. Incidence of bleeding symptoms in 100 patients with inherited afibrinogenemia or hypofibrinogenemia. $J$ Thromb Haemost. 2006;4(7):1634-1637.

13. Roque H, Stephenson C, Lee MJ, et al. Pregnancy-related thrombosis in a woman with congenital afibrinogenemia: a report of two successful pregnancies. Am J Hematol. 2004;76(3):267-270.

14. Parameswaran R, Dickinson JP, de LS, Keeling DM, Colvin BT Spontaneous intracranial bleeding in two patients with congenital afibrinogenaemia and the role of replacement therapy. Haemophilia 2000;6(6):705-708.

15. Henselmans JM, Meijer K, Haaxma R, Hew J, van der MJ. Recurrent spontaneous intracerebral hemorrhage in a congenitally afibrinogenemic patient: diagnostic pitfalls and therapeutic options. Stroke. 1999;30(11):2479-2482.

16. Menart C, Sprunck N, Duhaut P, et al. Recurrent spontaneous intracerebral hematoma in a patient with afibrinogenemia. Thromb Haemost. 1998;79(1):241-242.

17. Koussi A, Economou M, Athanasiou-Metaxa M. Intra-abdominal hemorrhage due to a ruptured corpus luteum cyst in a girl with congenital afibrinogenemia. Eur J Pediatr. 2001;160(3):196.

18. Castaman G, Ruggeri M, Rodeghiero F. Congenital afibrinogenemia: successful prevention of recurrent hemoperitoneum during ovulation by oral contraceptive. Am J Hematol. 1995;49(4):363-364.

19. Bottini E, Pareti FI, Mari D, et al. Prevention of hemoperitoneum during ovulation by oral contraceptives in women with type III von Willebrand disease and afibrinogenemia. Case reports. Haematologica. 1991;76(5):431-433.

20. Schneider D, Bukovsky I, Kaufman S, Sadovsky G, Caspi E. Severe ovarian hemorrhage in congenital afibrinogenemia. Acta Obstet Gynecol Scand. 1981;60(4):431.

21. de Moerloose P, Neerman-Arbez M. Treatment of congenital fibrinogen disorders. Expert Opin Biol Ther. 2008;8(7):979-992.

22. Fuchs RJ, Levin J, Tadel M, Merritt W. Perioperative coagulation management in a patient with afibrinogenemia undergoing liver transplantation. Liver Transpl. 2007;13(5):752-756.

23. Oruc N, Tokat Y, Killi R, Tombuloglu M, Ilter T. Budd-Chiari syndrome in an afibrinogenemic patient: a paradoxical complication. Dig Dis Sci. 2006;51(2):378-380.

24. Mosesson MW. Antithrombin I. Inhibition of thrombin generation in plasma by fibrin formation. Thromb Haemost. 2003;89(1):9-12.

25. de Bosch NB, Mosesson MW, Ruiz-Saez A, Echenagucia M, Rodriguez-Lemoin A. Inhibition of thrombin generation in plasma by fibrin formation (Antithrombin I). Thromb Haemost. 2002;88(2):253-258.
26. Korte W, Feldges A. Increased prothrombin activation in a patient with congenital afibrinogenemia is reversible by fibrinogen substitution. Clin Investig. 1994;72(5):396-398.

27. Remijn JA, Wu YP, Ijsseldijk MJ, et al. Absence of fibrinogen in afibrinogenemia results in large but loosely packed thrombi under flow conditions. Thromb Haemost. 2001;85(4):736-742.

28. Hanano M, Takahashi H, Itoh M, Shibata A. Coexistence of congenital afibrinogenemia and protein $\mathrm{C}$ deficiency in a patient. Am J Hematol. 1992;41(1):57-60.

29. Girard C, Guillot B, Biron C, et al. Digital skin necrosis in congenital afibrinogenaemia associated with hepatitis $\mathrm{C}$ virus infection, mixed cryoglobulinaemia and anticardiolipin antibodies. Acta Derm Venereol. 2005;85(1):56-59.

30. Kinebuchi A, Ohtsuka T, Ishida S, et al. Leg ulcer presenting in a patient with congenital afibrinogenaemia. Eur J Dermatol. 2002;12(1):70-72.

31. Rupec RA, Kind P, Ruzicka T. Cutaneous manifestations of congenital afibrinogenaemia. Br J Dermatol. 1996;134(3):548-550.

32. Shima M, Tanaka I, Sawamoto Y, et al. Successful treatment of two brothers with congenital afibrinogenemia for splenic rupture using heat- and solvent detergent-treated fibrinogen concentrates. $J$ Pediatr Hematol Oncol. 1997;19(5):462-465.

33. Fried K, Kaufman S. Congenital afibrinogenemia in 10 offspring of uncle-niece marriages. Clin Genet. 1980;17(3):223-227.

34. Ehmann WC, al-Mondhiry H. Congenital afibrinogenemia and splenic rupture. Am J Med. 1994;96(1):92-94.

35. Keeling D, Tait C, Makris M. Guideline on the selection and use of therapeutic products to treat haemophilia and other hereditary bleeding disorders. A United Kingdom Haemophilia Center Doctors' Organisation (UKHCDO) guideline approved by the British Committee for Standards in Haematology. Haemophilia. 2008;14(4):671-684.

36. Bolton-Maggs PH, Perry DJ, Chalmers EA, et al. The rare coagulation disorders - review with guidelines for management from the United Kingdom Haemophilia Centre Doctors' Organisation. Haemophilia. 2004;10(5):593-628.

37. Key NS, Negrier C. Coagulation factor concentrates: past, present, and future. Lancet. 2007;370(9585):439-448.

38. Chandra S, Groener A, Feldman F. Effectiveness of alternative treatments for reducing potential viral contaminants from plasma-derived products. Thromb Res. 2002;105(5):391-400.

39. Negrier C, Rothschild C, Goudemand J, et al. Pharmacokinetics and pharmacodynamics of a new highly secured fibrinogen concentrate. J Thromb Haemost. 2008;6(9):1494-1499.

40. Kreuz W, Meili E, Peter-Salonen K, et al. Pharmacokinetic properties of a pasteurised fibrinogen concentrate. Transfus Apher Sci. 2005;32(3):239-246.

41. Kreuz W, Meili E, Peter-Salonen K, et al. Efficacy and tolerability of a pasteurised human fibrinogen concentrate in patients with congenital fibrinogen deficiency. Transfus Apher Sci. 2005;32(3):247-253.

42. Haberer JP, Obstler C, Samama CM, et al. Postoperative deep venous thrombosis in a woman with congenital afibrinogenaemia treated with fibrinogen concentrates. Eur J Anaesthesiol. 2008;25(6):519-521.

43. De Mattia D, Regina G, Giordano P, et al. Association of congenital afibrinogenemia and $\mathrm{K}$-dependent protein $\mathrm{C}$ deficiency-a case report. Angiology. 1993;44(9):745-749.

44. Cronin C, Fitzpatrick D, Temperley I. Multiple pulmonary emboli in a patient with afibrinogenaemia. Acta Haematol. 1988;79(1):53-54.

45. Schuepbach RA, Meili EO, Schneider E, Peter U, Bachli EB. Lepirudin therapy for thrombotic complications in congenital afibrinogenaemia. Thromb Haemost. 2004;91(5):1044-1046.

46. Ra'anani P, Levi Y, Varon D, Gitel S, Martinowitz U. Congenital afibrinogenemia with bleeding, bone cysts and antibodies to fibrinogen. Harefuah. 1991;121(9):291-293.

47. De Vries A, Rosenbrg T, Kochwa S, Boss JH. Precipitating antifibrinogen antibody appearing after fibrinogen infusions in a patient with congenital afibrinogenemia. Am J Med. 1961;30:486-494. 
48. Ehmann WC, al-Mondhiry H. Splenic rupture in afibrinogenemia: conservative versus surgical management. Am J Med. 1995;99(4):444.

49. Aygoren-Pursun E, Martinez SI, Rusicke E, et al. Retrochorionic hematoma in congenital afibrinogenemia: resolution with fibrinogen concentrate infusions. Am J Hematol. 2007;82(4):317-320.

50. Rizk DE, Kumar RM. Congenital afibrinogenemia: treatment of excessive menstrual bleeding with continuous oral contraceptive. Am J Hematol. 1996;52(3):237-238.

51. MacKinnon HH, Fekete JF. Congenital afibrinogenemia. Vascular changes and multiple thromboses induced by fibrinogen infusions and contraceptive medication. Can Med Assoc J. 1971;104(7):597-599.

52. Neerman-Arbez M, Vu D, bu-Libdeh B, Bouchardy I, Morris MA. Prenatal diagnosis for congenital afibrinogenemia caused by a novel nonsense mutation in the $F G B$ gene in a Palestinian family. Blood. 2003;101(9):3492-3494.

53. Kobayashi T, Kanayama N, Tokunaga N, Asahina T, Terao T. Prenatal and peripartum management of congenital afibrinogenaemia. $\mathrm{Br} J$ Haematol. 2000;109(2):364-366.
54. Evron S, Anteby SO, Brzezinsky A, Samueloff A, Eldor A. Congenital afibrinogenemia and recurrent early abortion: a case report. Eur J Obstet Gynecol Reprod Biol. 1985;19(5):307-311.

55. James AH. More than menorrhagia: a review of the obstetric and gynaecological manifestations of bleeding disorders. Haemophilia. 2005;11(4):295-307.

56. de Moerloose P, Neerman-Arbez M. Congenital fibrinogen disorders. Semin Thromb Hemost. 2009;35(4):356-366.

57. Inamoto $Y$, Terao $T$. First report of case of congenital afibrinogenemia with successful delivery. Am J Obstet Gynecol. 1985;153(7):803-804.

58. Kobayashi T, Asahina T, Maehara K, et al. Congenital afibrinogenemia with successful delivery. Gynecol Obstet Invest. 1996;42(1):66-69.

59. Trehan AK, Fergusson IL. Congenital afibrinogenaemia and successful pregnancy outcome. Case report. Br J Obstet Gynaecol. 1991;98(7):722-724.

60. Peyvandi F, Duga S, Akhavan S, Mannucci PM. Rare coagulation deficiencies. Haemophilia. 2002;8(3):308-321.
Vascular Health and Risk Management

\section{Publish your work in this journal}

Vascular Health and Risk Management is an international, peerreviewed journal of therapeutics and risk management, focusing on concise rapid reporting of clinical studies on the processes involved in the maintenance of vascular health; the monitoring, prevention and treatment of vascular disease and its sequelae; and the involvement of

\section{Dovepress}

metabolic disorders, particularly diabetes. This journal is indexed on PubMed Central and MedLine. The manuscript management system is completely online and includes a very quick and fair peer-review system, which is all easy to use. Visit http://www.dovepress.com/ testimonials.php to read real quotes from published authors. 